\title{
Metastatic Renal Cell Carcinoma in the Paranasal Sinus: A Case Report and Literature Review
}

\author{
Sung Jae Heo, Tae Gyun Kwon ${ }^{2}$, and Jung-Soo Kim ${ }^{1}$ \\ ${ }^{1}$ Departments of Otorhinolaryngology-Head and Neck Surgery, ${ }^{2}$ Urology, School of Medicine, Kyungpook National University, \\ Daegu, Korea
}

부비동에 발생한 전이성 신세포암 증례보고 및 문헌고찰

허성재 ${ }^{1} \cdot$ 권태균 $^{2} \cdot$ 김정수 $^{1}$

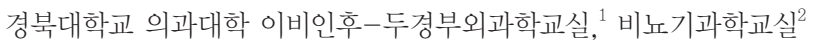

\author{
Received December 21, 2015 \\ Revised May 30, 2016 \\ Accepted June 8, 2016 \\ Address for correspondence \\ Jung-Soo Kim, MD \\ Department of Otorhinolaryngology- \\ Head and Neck Surgery, \\ School of Medicine, \\ Kyungpook National University, \\ 130 Dongdeok-ro, Jung-gu, \\ Daegu 41944, Korea \\ Tel $+82-53-420-5777$ \\ Fax $+82-53-423-4524$ \\ E-mail profsookim@gmail.com
}

\begin{abstract}
Metastatic renal cell carcinoma is very rare, especially in the paranasal sinus. Metastasis to multiple sites and late recurrence frequently occur in renal cell carcinoma. Moreover, typical treatment methods applied to this form of tumor have not produced a good response. Therefore, prognosis of patients with metastatic renal cell carcinoma is typically poor, and a consensus on appropriate treatment methods for these tumors has not been established. Recently, systemic immunotherapy and target therapy were employed to treat the tumor, but they have not been considered as definite treatment methods for metastatic renal cell carcinoma. Since paranasal sinus is surrounded by vital organs, choosing a treatment modality for a tumor in the paranasal sinus is more difficult than in other sites. We present the case of metastatic renal cell carcinoma in the paranasal sinus that was successfully treated by endoscopic surgery with a literature review about treatment methods.

Korean J Otorhinolaryngol-Head Neck Surg 2016;59(12):877-81
\end{abstract}

Key Words Metastasis - Paranasal sinus · Renal cell carcinoma.

\section{Introduction}

Renal cell carcinoma (RCC), which is the most common malignant tumor of the kidney, is more prevalent in men aged 30 to 60 years. ${ }^{1)}$ Up to $30 \%$ of patients with RCC present with metastasis; the lungs (75\%), regional lymph nodes (65\%), and bone and liver (40\%) are common sites, and $15 \%$ of patients have metastasis in the head and neck area. ${ }^{1,2)}$ The thyroid gland is the most common site for tumor metastasis among the head and neck areas, ${ }^{3)}$ whereas the paranasal sinus is a rare metastatic site of RCC.

Due to its rarity, only a few cases of an RCC metastatic tumor in the paranasal sinus have been reported. As such, a consensus on treatment strategy has not been established. Surgery is generally considered as the first line treatment in the case of small, solitary metastasis; however, in the cases of multiple metastases or extensive disease with local spread, the role of surgery seems to be limited, and debulking is often recommended. ${ }^{4)}$ Moreover, the effectiveness of radiotherapy and chemotherapy has not proven to be acceptable for metastatic RCC. ${ }^{3)}$ Recently, systemic immunotherapy (including interleukin-2 and interferon $\alpha$ ) and target therapy (sunitinib malate, an inhibitor of tyrosine kinases) are frequently employed to manage the metastatic RCC. ${ }^{5)}$ However, their roles and appropriate indications have not yet been defined. Therefore, numerous surgeons encounter a difficulty in selecting the most suitable modality for the treatment of metastatic RCC.

The excision of solitary metastatic RCC after control of renal lesion shows a $41 \%$ survival rate at 2 years and $13 \%$ at 5 
years. ${ }^{6)}$ Patients with multiple metastases show poorer overall prognosis; ranging from 0 to $7 \%$ in the 5 -year survival rates. ${ }^{7)}$ Although RCC with multiple metastases appears to have poor prognosis, the presenting patient was successfully treated with endoscopic surgery for paranasal sinus metastasis and with sunitinib malate for lung metastasis. Therefore, we present a case of metastatic RCC involving the ethmoid and sphenoid sinuses with lung metastasis in aspect of treatment modality with a pertinent review of literature.

\section{Case}

A 67-year-old man visited a private otolaryngology clinic with a complaint of left nasal obstruction that had persisted for 3 weeks. He was referred to our institution as a mass not characteristic of a polyp was found in the left nasal cavity. In the past, the patient had undergone a laparoscopic radical nephrectomy of the right kidney 10 years prior, with a diagnosis of RCC $(7.6 \times 5.5 \mathrm{~cm}$, clear cell type, T2N0M0 Fuhrman grade II). Five years after the RCC surgery, multiple metastatic nodules in both lungs were found, and the patient was treated with three cycles of chemotherapy consisting of gemicitabine and cisplatin. The metastatic nodules did not respond to the chemotherapy, but had maintained a stable state for the five years prior to his entry into our institution.

Upon endoscopic examination performed at our clinic, an

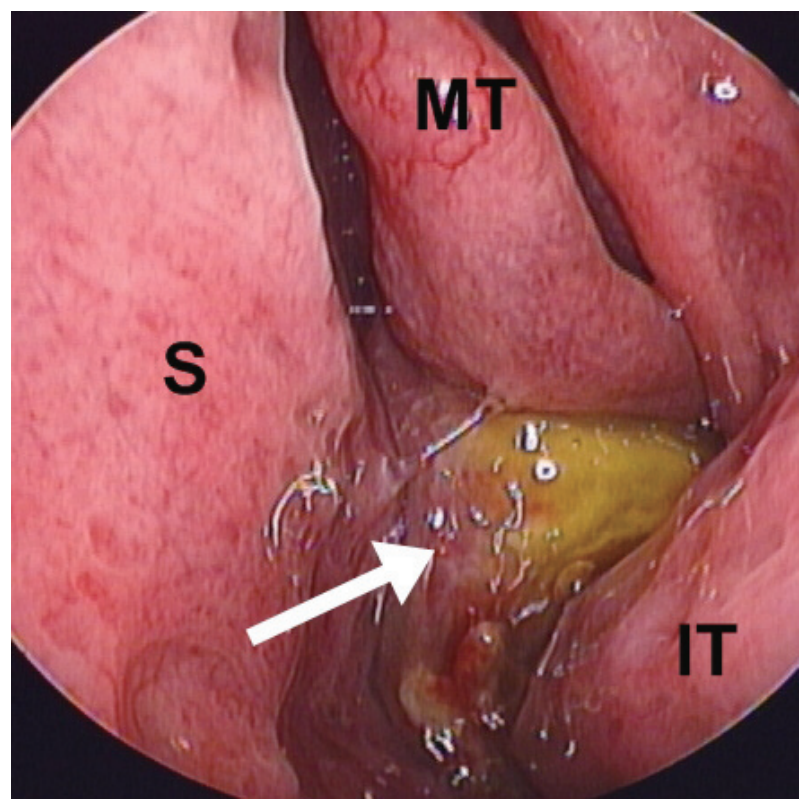

Fig. 1. On the endoscopic examination of left nasal cavity, a reddish mass (white line arrow) with prominent vessels on the irregular surface is shown between the septum and turbinates. S: septum, MT: middle turbinate, IT: inferior turbinate. irregular and reddish tumoral lesion was found among the inferior turbinate, middle turbinate, and nasal septum (Fig. 1). Paranasal sinus computed tomography (PNS CT) revealed an enhanced $5 \times 4 \mathrm{~cm}$ sized mass in the ethmoid sinus extending to the sphenoid sinus (Fig. 2A and B). The mass eroded the skull base, medial wall of orbit, inferior and middle turbinates, and nasal septum. Endoscopic biopsy was performed and
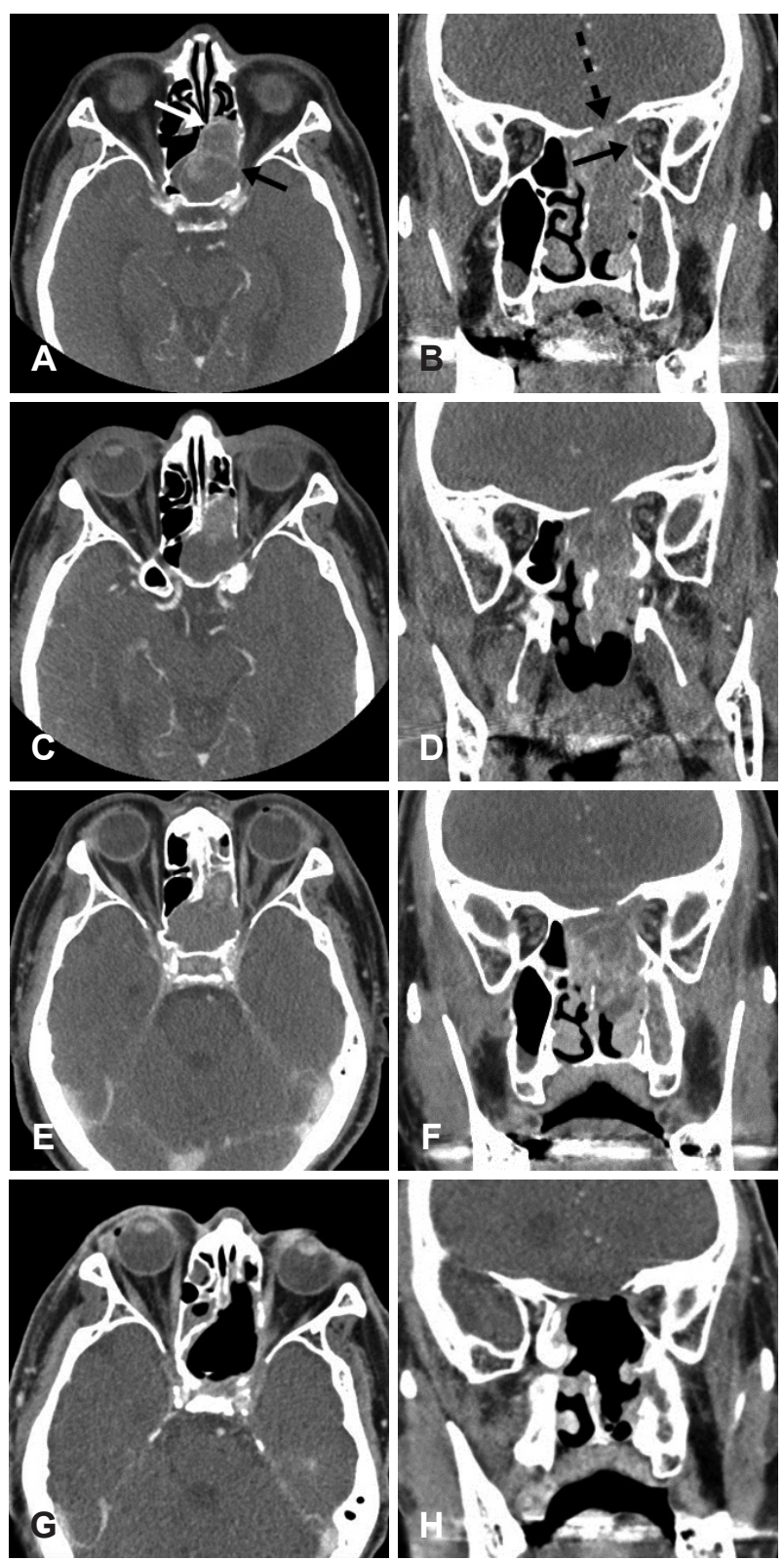

Fig. 2. Computed tomography of paranasal sinus with contrast. In the initial axial and coronal images (A and $B)$, the mass occupied nasal cavity, ethmoid sinus, and sphenoid sinus and destructed lateral lamella of the cribriform plate (white line arrow) and medial bony wall of orbit (black line arrow), and invaded skull base (black dotted line arrow). The size of mass was not changed after sunitinib malate treatment $(C$ and $D)$ and even after radiation therapy ( $E$ and $F$ ). The mass was completely removed by surgery ( $G$ and $H$ ). 

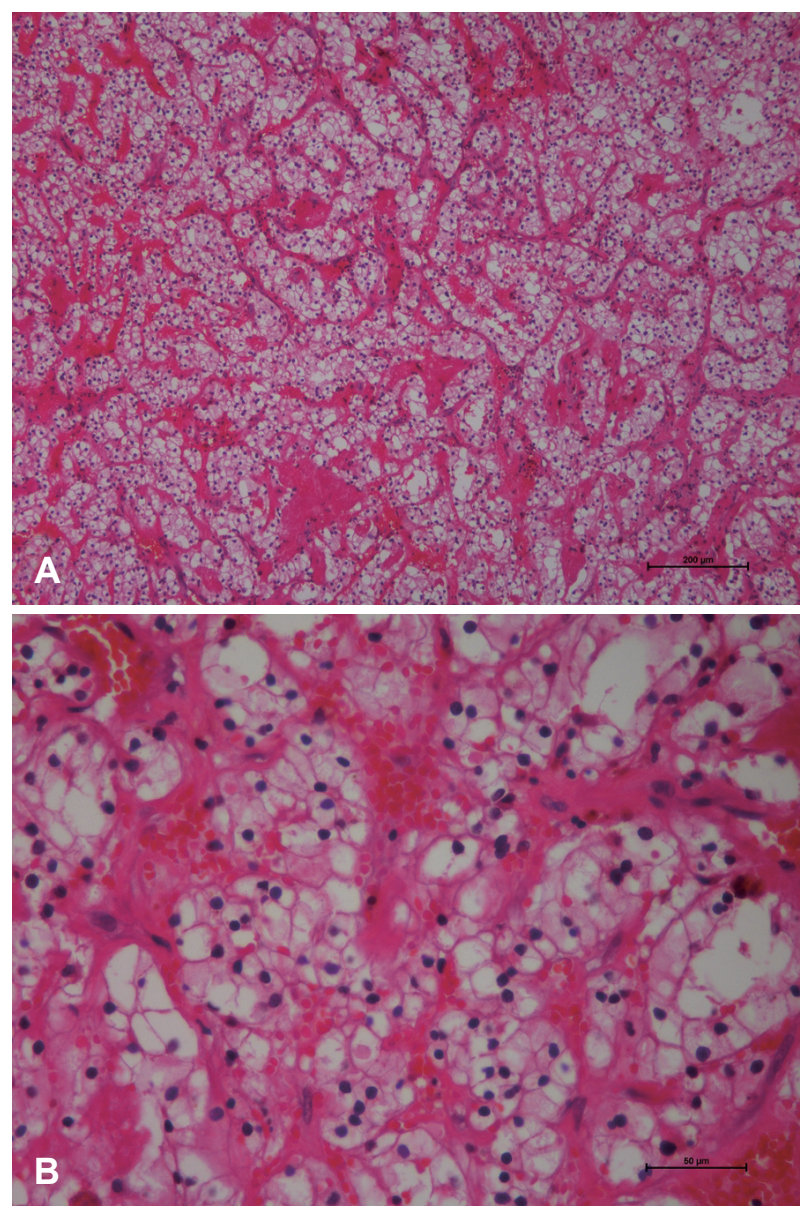

Fig. 3. Histopathologic findings of the metastatic renal cell carcinoma in the paranasal sinus show polygonal cells with typical clear cytoplasms (hematoxyline-eosin; magnification, $\times 100$ in $\mathrm{A}$ and $\times 400$ in B).

histopathological examination diagnosed the mass as metastatic RCC (Fig. 3).

Target therapy (sunitinib malate) was employed to the patient as the first line treatment method. After applying sunitinib malate (25-50 mg daily for 6 months), the size and number of metastatic lung nodules decreased, but the mass in the paranasal sinus was unchanged (Fig. $2 \mathrm{C}$ and D). In the next treatment modality, radiotherapy (5400 cGy) was applied to the lesion in the paranasal sinus. On a follow-up PNS CT after the radiotherapy, necrosis was confirmed inside of the mass; however, it did not show any change in size (Fig. 2E and F). Therefore, we decided to remove the mass surgically as a final treatment option.

Endoscopic surgery was conducted under general anesthesia. The mass was found to originate in the anterior and inferior wall of sphenoid sinus and extended to the anterior ethmoid and sphonoid sinuses, skull base, and medial wall of orbit. The mass was completely removed with a $0.5-1 \mathrm{~cm}$ safety margin, excluding the medial wall of orbit and skull base (Fig. 2G and H). Fortunately, dura and periosteum were not invaded by the tumor, so these tissues were preserved. Bleeding occurred, even with a gentle touch of the mass, but massive bleeding did not occur during surgery. Consequently, serious problems such as intracranial and intraorbital complications and hypovolemic shock did not occur.

After the surgery, the recurrence of the nasal mass was not observed and the patient continued sunitinib malate (25$37.5 \mathrm{mg}$ daily) treatment for metastatic lung nodules. Likewise prior to the surgery, the lung lesions had responded to the sunitinib malate and disappeared after a year. On the 46th month of follow-up, recurrence has not been found in the nasal cavity or the lung.

\section{Discussion}

Paranasal sinus is a rare metastatic site for RCC. This metastasis is thought to occur via hematogenous spread. One possible route is via inferior vena cava spread to the lung and then carotid arterial systems. ${ }^{8)}$ The other possible theory is retrograde movement of tumor cells via the Baston sacral venous plexus to the head and neck, bypassing the lungs. ${ }^{6)}$ Since the patient showed lung metastases, inferior vena cava is seems to be the spread route in this case.

Patients undergoing a complete resection of RCC frequently encounter not only late metastasis, but also late recurrence even 10 years after successful management. ${ }^{9)}$ Moreover, since the 5-year survival rate of patients with RCC metastasis is around $10 \%$ and multiple metastases are common, planning a treatment strategy for RCC metastasis is difficult. ${ }^{3)}$

To our knowledge, 47 cases of nasal metastasis of RCC have been reported in the literature. We reviewed and analyzed these to decide on the appropriate treatment modality for the case of RCC metastasis in the paranasal sinus that presented at our clinic. We excluded cases of RCC metastasis to the septum and external nose (21 cases), as this treatment strategy is quite different from metastasis to paranasal sinus. A mass in the septum and external nose is relatively easy to remove because vital organs (such as the orbit and brain) are usually distant from the mass. In contrast, excision of tumor in the paranasal sinus usually accompanies a risk of intracranial or intraorbital complications. Analyses of cases of metastases to paranasal sinus (27 cases) are presented in Table 1.

Male patients and patients with old age were predominant in these cases, and epistaxis was most common symptom of 
Table 1. Clinical data of metastatic renal cell carcinoma in the paranasal sinus $(n=27)$

\begin{tabular}{lc}
\hline \multicolumn{1}{c}{ Variables } & No. (\% or range) \\
\hline Sex, male/female & $21 / 6$ \\
Mean age (years) & $59.19(35-80)$ \\
Symptom & \\
Epistaxis & $13(48.1 \%)$ \\
Nasal obstruction & $6(22.2 \%)$ \\
Others* & $8(29.6 \%)$ \\
Involved sinus & \\
Maxillary sinus & $4(14.8 \%)$ \\
Ethmoid sinus & $6(22.2 \%)$ \\
Sphenoid sinus & $3(11.1 \%)$ \\
Multiple sinuses & $14(51.9 \%)$ \\
Mean size of metastatic tumor (cm) & $4.17(3.0-6.0)$ \\
Treatment & \\
Surgery & $13(48.1 \%)$ \\
Radiation & $9(33.3 \%)$ \\
Immunotherapy or target therapy & $3(11.1 \%)$ \\
No treatment & $2(7.4 \%)$ \\
Mean period of follow-up (months) & $19(0-78)$ \\
Prognosis & \\
Remission & $5(18.5 \%)$ \\
Stable & $10(37.0 \%)$ \\
Recurrence & $2(7.4 \%)$ \\
Expiration & $3(11.1 \%)$ \\
\hline
\end{tabular}

*others include facial swelling, incidental finding, proptosis, diplopia, and hyposmia, ${ }^{\dagger}$ prognosis is a result of treatments during mean 19 months follow-up (range, 0-78)

RCC metastasis in the paranasal sinus. The metastasis was found at mean of 6.46 years after kidney resection (range, 0-17 years); 10 cases (37\%) showed metastasis 10 years after nephrectomy. Multiple metastases occurred in 6 cases $(22.2 \%$; 4 cases in the lung, 1 case in the thyroid and 1 in the pharynx, respectively). Three patients had orbital complications and 6 patients showed both orbital and intracranial complications. Bone erosions occurred in 16 cases (59.3\%) and the mean size of the metastatic tumor was $4.17 \mathrm{~cm}$ (range, 3-6 cm). Endoscopic biopsy (19 cases, 70.4\%) was a frequently conducted method for diagnosis, followed by fine-needle aspiration. Nine patients (33.3\%) underwent embolization for a control of epistaxis or prevention of massive bleeding during operation.

Surgery was the leading treatment method (13 patients, $48.1 \%$ ) followed by radiation therapy (33.3\%). Seven of the 13 patients received adjuvant irradiation before or after surgery. Two patients received chemotherapy or target therapy, and 4 patients received no additional treatment excluding surgery. Nine of the surgery patients showed favorable treatment re- sults ( 2 patients with remission; 7 patients with stable status), whereas 4 patients showed unfavorable or unknown results (1 patient with recurrence; 1 patient died; 2 patients with a loss of follow-up). Endoscopic surgery (5 patients) was the most common surgical method, while others underwent external approach methods ( 3 patients received craniofacial resection, 3 patients received total maxillectomy, 2 patients received rhinotomy). Among the nine patients who were administered radiation therapy, 3 patients achieved remission and 3 patients achieved a stable state. One patient died and 4 patients lost follow-up communication.

RCC is a resistant tumor for radiation therapy, ${ }^{10)}$ but radiation has shown acceptable treatment results for metastatic RCC. ${ }^{11)}$ On the literature analysis, radiation therapy was an important method for managing metastatic RCC as both a main and supplementary treatment. However, this case did not respond to the radiotherapy. Although radiation was not useful for removing the tumor in the present case, it may help reduce bleeding during surgery by means of inducing devascularization of the lesion.

Since RCC is highly resistant to chemotherapy, interleukin-2 and interferon- $\alpha$ have been employed as an alternative to chemotherapy. ${ }^{2)}$ However, response rate with the cytokines were poor (5 to $20 \%$ ) and median survival was 12 months. ${ }^{12)}$ Recently, sunitinib malate, an antiangiogenic agent, showed a favorable tumor response. ${ }^{13)}$ Motzer et al. ${ }^{2)}$ reported that progression-free survival was longer and response rates were higher in patients with metastatic RCC who received sunitinib malate than interferon- $\alpha$. However, in the present case, the metastatic RCC responded to sunitinib malate in the lung, but not the paranasal sinus.

Surgical resection of RCC metastasis in the paranasal sinus is advocated with reference to morbidity and quality of life. ${ }^{3)}$ Endoscopic surgery showed a low morbidity and contributed to manage the complaining symptoms such as epistaxis and nasal obstruction. On our analysis, surgery has shown positive results compared to other treatment modalities and was the only effective treatment method in this case. Although the present case had multiple metastases, complete surgical resection of the tumor was attributed to the successful control of the disease. Consequently, surgical treatment may be a good treatment option for metastatic RCC in the paranasal sinus, even in patients with multiple metastases or invasion to the skull base or orbital wall. 


\section{REFERENCES}

1) Nason R, Carrau RL. Metastatic renal cell carcinoma to the nasal cavity. Am J Otolaryngol 2004;25(1):54-7.

2) Motzer RJ, Hutson TE, Tomczak P, Michaelson MD, Bukowski RM, Rixe O, et al. Sunitinib versus interferon alfa in metastatic renal-cell carcinoma. N Engl J Med 2007;356(2):115-24.

3) Doğan S, Can IH, Sayn M, Ozer E, Bayz U, Yazici G, et al. The nasal septum: an unusual presentation of metastatic renal cell carcinoma. J Craniofac Surg 2009;20(4):1204-6.

4) Ziari M, Shen S, Amato RJ, Teh BS. Metastatic renal cell carcinoma to the nose and ethmoid sinus. Urology 2006;67(1):199.

5) Simo R, Sykes AJ, Hargreaves SP, Axon PR, Birzgalis AR, Slevin NJ, et al. Metastatic renal cell carcinoma to the nose and paranasal sinuses. Head Neck 2000;22(7):722-7.

6) Gottlieb MD, Roland JT Jr. Paradoxical spread of renal cell carcinoma to the head and neck. Laryngoscope 1998;108(9):1301-5.

7) Hefer T, Joachims HZ, Golz A. Metastatic renal cell carcinoma to the nose. Eur Arch Otorhinolaryngol 1994;251(2):127-9.

8) Nahum AM, Bailey BJ. Malignant tumors metastatic to the paranasal sinuses: case report and review of the literature. Laryngoscope 1963; 73:942-53.

9) Hong SL, Jung DW, Roh HJ, Cho KS. Metastatic renal cell carcinoma of the posterior nasal septum as the first presentation 10 years after nephrectomy. J Oral Maxillofac Surg 2013;71(10):1813.el-7.

10) Skinner DG, Colvin RB, Vermillion CD, Pfister RC, Leadbetter WF. Diagnosis and management of renal cell carcinoma. A clinical and pathologic study of 309 cases. Cancer 1971;28(5):1165-77.

11) DiBiase SJ, Valicenti RK, Schultz D, Xie Y, Gomella LG, Corn BW. Palliative irradiation for focally symptomatic metastatic renal cell carcinoma: support for dose escalation based on a biological model. J Urol 1997;158(3 Pt 1):746-9.

12) Negrier S, Escudier B, Lasset C, Douillard JY, Savary J, Chevreau $C$, et al. Recombinant human interleukin-2, recombinant human interferon alfa-2a, or both in metastatic renal-cell carcinoma. Groupe Français d'Immunothérapie. N Engl J Med 1998;338(18):1272-8.

13) Motzer RJ, Rini BI, Bukowski RM, Curti BD, George DJ, Hudes GR, et al. Sunitinib in patients with metastatic renal cell carcinoma. JAMA 2006;295(21):2516-24. 